\title{
Electrophysiological Correlates of Word Retrieval in Traumatic Brain Injury
}

\author{
Julie M. Fratantoni, ${ }^{1}$ Bambi L. DeLaRosa, ${ }^{1}$ Nyaz Didehbani, ${ }^{1,2}$ John Hart Jr, ${ }^{1,3}$ and Michael A Kraut ${ }^{4}$
}

\begin{abstract}
Persons who have had a traumatic brain injury (TBI) often have word retrieval deficits; however, the underlying neural mechanisms of such deficits are yet to be clarified. Previous studies in normal subjects have shown that during a word retrieval task, there is a $750 \mathrm{msec}$ event-related potential (ERP) divergence detected at the left fronto-temporal region when subjects evaluate word pairs that facilitate retrieval compared with responses elicited by word pairs that do not facilitate retrieval. In this study, we investigated the neurophysiological correlates of word retrieval networks in 19 retired professional athletes with TBI and 19 healthy control (HC) subjects. We recorded electroencephalography (EEG) in the participants during a semantic object retrieval task. In this task, participants indicated whether presented word pairs did (retrieval) or did not (non-retrieval) facilitate the retrieval of an object name. There were no significant differences in accuracy or reaction time between the two groups. The EEG showed a significant group by condition interaction over the left fronto-temporal region. The HC group mean amplitudes were significantly different between conditions, but the TBI group data did not show this difference, suggesting neurophysiological effects of injury. These findings provide evidence that ERP amplitudes may be used as a marker of disrupted semantic retrieval circuits in persons with TBI even when those persons perform normally.
\end{abstract}

Keywords: EEG; ERP; sports concussion; traumatic brain injury; word retrieval

\section{Introduction}

$\mathbf{T}$ RAUMATIC BRAIN INJURY (TBI) can affect a person's ability to successfully perform activities of daily living. ${ }^{1}$ One of the primary symptoms after TBI is impaired word retrieval. ${ }^{2}$ Word retrieval is the "process of finding the correct terminology for an object, picture, orthographic representation, or conversation in which a person converts the initial conception to a lexical version." 3 Word retrieval is a pervasive complaint among athletes with TBI. Studies have shown that persons with TBI demonstrate decreased efficiency in the ability to access feature information in the presence of intact semantic knowledge. ${ }^{4}$ The inability to retrieve and integrate features can interfere with activation of a semantic memory and its associated word representation. Efficient word retrieval is necessary for most high-level tasks in the workplace, so even mild deficits can have a deleterious effect on communication in daily life.

Word retrieval requires precise timing of interactions between brain regions. ${ }^{5}$ Electroencephalography (EEG) has high temporal resolution and is sensitive to the timing of neuronal synaptic and dendritic activity. ${ }^{6}$ Studies have shown alteration in event-related potentials (ERP) after TBI, and thus ERP may be useful as markers to define specific treatment targets. ${ }^{7-9}$ ERP abnormalities in persons with TBI have been described in several cognitive domains including visual information processing ${ }^{10}$ and visual working memory. ${ }^{11}$ Although markers for semantic word meaning have been suggested, ${ }^{12}$ a specific marker for word retrieval is currently not well established.

The cognitive processes associated with word retrieval are complex and not fully understood. To study the mechanisms of word retrieval, our group has utilized an EEG Semantic Object Retrieval Test (SORT) in various populations. ${ }^{13-16}$ The SORT task uses word pairs as stimuli, and the participant indicates whether the word pair elicits the retrieval of an object name (retrieval condition) or does not elicit retrieval (non-retrieval condition). Healthy adults demonstrate an ERP amplitude divergence starting at approximately $750 \mathrm{msec}$ post-stimulus in the left fronto-temporal regions when comparing retrieval and non-retrieval word pairs. ${ }^{15}$ Previous

\footnotetext{
${ }^{1}$ Center for BrainHealth, The University of Texas at Dallas, Dallas, Texas.

${ }^{2}$ Departments of Psychiatry and ${ }^{3}$ Neurology and Neurotherapeutics, The University of Texas Southwestern Medical Center, Dallas, Texas.

${ }^{4}$ Department of Radiology, The Johns Hopkins University School of Medicine, Baltimore, Maryland.

(c) Julie M. Fratantoni, et al., 2016; Published by Mary Ann Liebert, Inc. This Open Access article is distributed under the terms of the Creative Commons Attribution Noncommercial License (http://creativecommons.org/licenses/by-nc/4.0/) which permits any noncommercial use, distribution, and reproduction in any medium, provided the original author(s) and the source are credited.
} 
studies of disordered populations found that condition-related differences in ERP amplitude on the SORT task correlated with memory deficits. ${ }^{16,17}$

Taken together, these findings indicate that there are different patterns of neuronal activation during successful retrieval of a word as opposed to non-retrieval. In addition to its role in SORT, the left fronto-temporal area is also of interest because it plays an important role in language production. ${ }^{18,19}$ Thus, using the SORT task to study word retrieval in patients with a history of TBI may help clarify further the level of cognitive disruption in these persons.

We chose to study retired professional athletes because of their increased risk of multiple TBIs and increased incidence of associated word finding problems. Our objectives were to evaluate behavioral performance of word retrieval using the SORT task and to characterize ERP differences between a group of individuals with a history of TBI and a group of healthy control (HC) subjects. We expected to find differences in ERP amplitudes in the left fronto-temporal region in the TBI group compared with the HC group. Comparing the electrophysiological and behavioral changes in these groups will allow us to assess whether ERP for semantic retrieval is a useful marker of dysfunction in repeated head trauma.

\section{METHODS}

\section{Subjects}

TBI participants were recruited from local retired National Football League (NFL) players living in the North Texas region, at a meeting of the NFL Players Association local chapter, local advertising, and by word of mouth. Control participants were not professional athletes. All participants were male, native English speakers. There were a total of 38 subjects (19 TBI). Demographic information is listed in Table 1. Participants completed a questionnaire that inquired about history of head injury. Athletes reported TBI ranging from mild to severe. TBI history information is listed in Table 2. Control participants were excluded if they endorsed a history of head injury. To focus on the effects of TBI without co-morbidity, participants with mild cognitive impairment (MCI) were excluded from the current analysis.

An independent sample $t$ test revealed a significant difference for age between the TBI group $(\mathrm{M}=50.0, \mathrm{SD}=13.1)$ and $\mathrm{HC}$ group $(\mathrm{M}=59.6, \mathrm{SD}=13.9) ; t(36)=2.2, p=0.034$. Thus, all analyses used age as a covariate to control for group differences. Informed consent was obtained from all participants in accordance with the protocols approved by the Institutional Review Boards of the University of Texas at Dallas and of the University of Texas Southwestern Medical Center.

Table 1. Participant Demographics

\begin{tabular}{|c|c|c|c|c|c|}
\hline Group & $N$ & Age & $\begin{array}{l}\text { Years of } \\
\text { education }\end{array}$ & $I Q$ & Race \\
\hline $\mathrm{HC}$ & 19 & $\begin{array}{c}M=59.6 \\
(\mathrm{SD}=13.9)\end{array}$ & $\begin{array}{c}\mathrm{M}=16.2 \\
(\mathrm{SD}=2.0)\end{array}$ & $\begin{array}{l}\mathrm{M}=117.2^{\mathrm{a}} \\
(\mathrm{SD}=9.2)\end{array}$ & $\begin{array}{r}\text { 1/18 African } \\
\text { American/ } \\
\text { Caucasian }\end{array}$ \\
\hline TBI & 19 & $\begin{array}{c}\mathrm{M}=50.0 \\
(\mathrm{SD}=13.1)\end{array}$ & $\begin{array}{l}M=16.4^{\mathrm{a}} \\
(\mathrm{SD}=1.0)\end{array}$ & $\begin{array}{l}\mathrm{M}=109.8^{\mathrm{a}} \\
(\mathrm{SD}=9.1)\end{array}$ & $\begin{array}{c}\text { 7/12 African } \\
\text { Americans/ } \\
\text { Caucasian }\end{array}$ \\
\hline
\end{tabular}

IQ, intelligence quotient; HC, healthy control subjects; SD, standard deviation; TBI, traumatic brain injury.

${ }^{\mathrm{a}} \mathrm{N}, 18$.
Table 2. Participant Traumatic Brain Injury History

\begin{tabular}{|c|c|c|c|}
\hline & Mean & $S D$ & Range \\
\hline Total number of concussions & 8.3 & 5.4 & $1-20$ \\
\hline Number of grade 3 concussions & 1.7 & 1.7 & $1-5$ \\
\hline Years since retired ${ }^{\mathrm{a}}$ & 23.2 & 14.4 & $5-50$ \\
\hline
\end{tabular}

$\mathrm{SD}$, standard deviation

$\mathrm{N}, 18$

${ }^{\mathrm{a}} \mathrm{N}, 17$

\section{General procedure}

Scalp EEG data were recorded while subjects performed the SORT task. Participants were seated approximately 46 inches away from a computer monitor. The participant was presented with two visual words representing object features. In some instances, the word pairs facilitated retrieval (i.e., "desert" and "humps" should elicit "camel") and in other trials, word pairs did not (i.e., "desert", and "barks"). The stimuli consisted of 57 retrieval pairs of words and 55 non-retrieval pairs. The words were presented one above the other. Both words remained on the screen for $3 \mathrm{sec}$. In between each pair of words, a fixation cross was displayed on the screen for $3 \mathrm{sec}$. Subjects used a button box with their right hand and were instructed to press the button under their index finger for retrievals and the button under their middle finger for non-retrievals.

\section{ERP acquisition and data analysis}

We recorded EEG using a 64-electrode Neuroscan Quickcap, Neuroscan SynAmps2 amplifiers, and Scan 4.3.2 software. Continuous EEG was sampled at $1 \mathrm{kHz}$. Electrode impedances were kept below $5 \mathrm{kOhms}$. The data were high-pass filtered offline at $1 \mathrm{~Hz}$ and low pass filtered at $30 \mathrm{~Hz}$. The reference electrode was at the vertex of the scalp.

We analyzed the data using functions from EEGLAB version $13-1-1 b^{20}$ running under Matlab 7.11.0 (MATLAB and EEGLAB Toolbox Release 2010b, The MathWorks, Inc., Natick, MA). Preprocessing consisted of down-sampling to $512 \mathrm{~Hz}$, excluding the data from electrodes that exhibited unacceptably high levels of electrical noise and correcting for artifacts using the "Runica" algorithm (with the "extended," 1 option), ${ }^{20,21}$ an implementation of the logistic infomax independent component analysis algorithm of Bell and Sejnowski. ${ }^{22}$

We identified stereotyped artifacts by visual inspection of the spatial and temporal representation of the independent components. Continuous data were then segmented into non-overlapping epochs spanning from $500 \mathrm{msec}$ before to $2000 \mathrm{msec}$ after the presentation of the visual stimuli. Epochs containing high amplitude, high frequency muscle noise and other irregular artifacts were removed. Only trials to which the subject responded correctly and those without artifacts were subjected to further analysis, rejecting on average $13 \%$ or less of all epochs. Data from missing electrodes were interpolated and re-referenced to the average reference. $^{23}$

For each trial and electrode, the mean amplitude of the prestimulus interval $(-100 \mathrm{msec}$ to $0 \mathrm{msec})$ was subtracted from each time point in the post-stimulus interval $(1 \mathrm{msec}$ to $1500 \mathrm{msec})$. Baseline-correction single trials were averaged within condition to estimate the ERP in each subject and condition (retrieval, nonretrieval). Based on previous word retrieval findings, we focused on 
Table 3. Behavioral Results of Electroencephalography TASK Performance

\begin{tabular}{lcc}
\hline & $\begin{array}{c}\text { TBI } \\
\text { (total } \mathrm{n}=19)\end{array}$ & $\begin{array}{c}H C \\
\text { (total } \mathrm{n}=19)\end{array}$ \\
\hline R-accuracy (\%) & $74.6(13.3)$ & $82.8(10.6)$ \\
NR-accuracy (\%) & $82.1(16.2)$ & $87.2(11.0)$ \\
R-RT (msec) & $1165.9(275.5)$ & $1087.9(152.2)$ \\
NR-RT (msec) & $1488.0(476.3)$ & $1376.1(295.1)$ \\
\hline
\end{tabular}

TBI, traumatic brain injury; $\mathrm{HC}$, health control subjects; $\mathrm{R}$, retrieval trials; NR, non-retrieval trials.

Each represents group mean (standard deviation).

the left fronto-temporal area (electrode F7). The mean amplitude at F7 was averaged during the time window of $750 \mathrm{msec}$ to $1000 \mathrm{msec}^{15,24}$

\section{Statistical analysis}

Reaction time and accuracy were analyzed separately using a mixed effect two-way analysis of variance (ANOVA) with age as a covariate (between-subject factor: group [TBI vs. HC]; withinsubject factor: condition [retrieval vs. non-retrieval]). A paired samples $t$ test was used to compare conditions (retrieval vs. nonretrieval). We ran an independent samples $t$ test to compare groups (TBI vs. HC) for both retrieval and non-retrieval conditions. All statistical analyses of behavioral data were performed using IBM Statistics 21 (IBM Corp. Released 2012. IBM SPSS Statistics for Windows, Version 21.0. Armonk, NY: IBM Corp.).

We tested for group difference and interaction by performing a mixed effect two-way ANOVA in IBM SPSS Statistics 21, with age as a covariate (between-subject factor: group [TBI vs. HC]; withinsubject factor: condition [retrieval; vs. non-retrieval]) on mean amplitude. The differences between retrieval and non-retrieval trials were analyzed using paired $t$ tests in the TBI versus HC groups. The differences between groups (TBI vs. HC) were analyzed using independent sample $t$ tests for both retrieval and nonretrieval conditions.

\section{Results}

\section{Behavioral results}

The behavioral results are summarized in Table 3 . We conducted an independent samples $t$ test to compare the TBI group and HC group mean reaction time and percent correct for retrieval and nonretrieval conditions. There was not a significant difference between groups for reaction time in retrieval $(t(36)=-1.39, p=0.174)$ or non-retrieval $(t(36)=-1.14, p=0.261)$. There was not a significant difference between groups for percent correct in retrieval $(t(36)=1.89, p=0.067)$ or non-retrieval $(t(36)=1.10, p=0.277)$. There was no group by condition interaction in either reaction time or accuracy $(p>0.1)$.

For each condition, both reaction time and percent correct were analyzed using a paired samples $t$ test. The mean accuracy for the retrieval condition was significantly lower $(\mathrm{M}=79.2 \%, \mathrm{SD}=12.4)$ than for the non-retrieval condition $(\mathrm{M}=84.7 \%, \mathrm{SD}=14.2 ; t(37)=$ $-2.46 ; p=.019)$. Mean reaction time latency for the retrieval condition was significantly shorter $(M=1136.91 \mathrm{msec}, \mathrm{SD}=220.6 \mathrm{msec})$ than the non-retrieval condition $(\mathrm{M}=1448.4 \mathrm{msec}, \mathrm{SD}=391.6 \mathrm{msec}$; $t(37)=-6.67 ; p<0.001)$. Both groups had longer reaction times and higher accuracy for the non-retrieval condition.

\section{EEG/ERP results}

The group average ERPs are depicted in Figure 1. The two-way ANOVA showed that for the mean amplitude of F7 between 750 and $1000 \mathrm{msec}$ post-stimulus, there was a significant group by condition interaction $F(1,35)=6.05, p=0.019$. A paired samples $t$ test was conducted to compare retrieval and non-retrieval conditions. There was a significant difference between the retrieval condition $(\mathrm{M}=.25 \mu \mathrm{V}, \mathrm{SD}=.41)$ and the non-retrieval condition $(\mathrm{M}=-.12 \mu \mathrm{V}, \mathrm{SD}=.38 ; t(18)=2.83 ; p=0.011)$ for the $\mathrm{HC}$ group. No significant difference was found between conditions for the TBI group (retrieval $\mathrm{M}=.12 \mu \mathrm{V}, \mathrm{SD}=.54$, non-retrieval $\mathrm{M}=.19 \mu \mathrm{V}, \mathrm{SD}=.51, t(18)=-.49 ; p=0.631)$.

An independent samples $t$ test revealed a significant difference between the TBI group $(\mathrm{M}=.19 \mu \mathrm{V}, \mathrm{SD}=.5)$ and the $\mathrm{HC}$ group

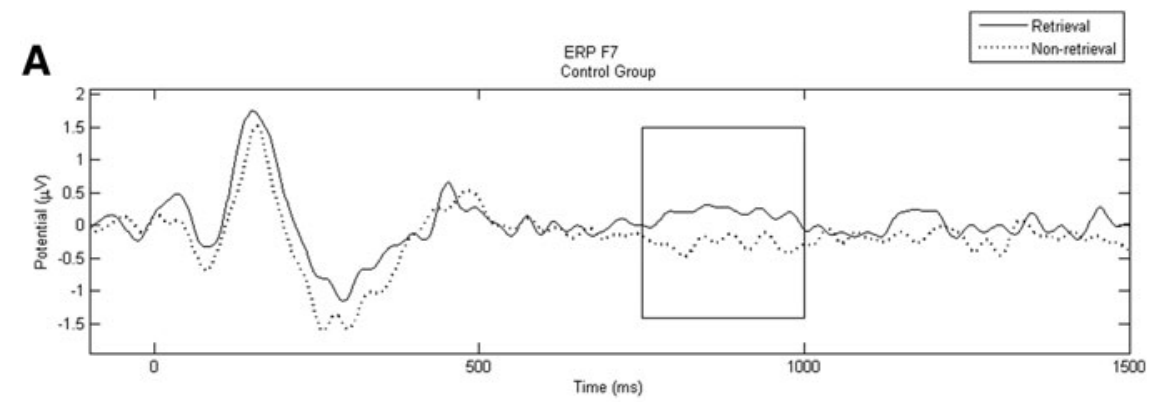

B

TBI Group

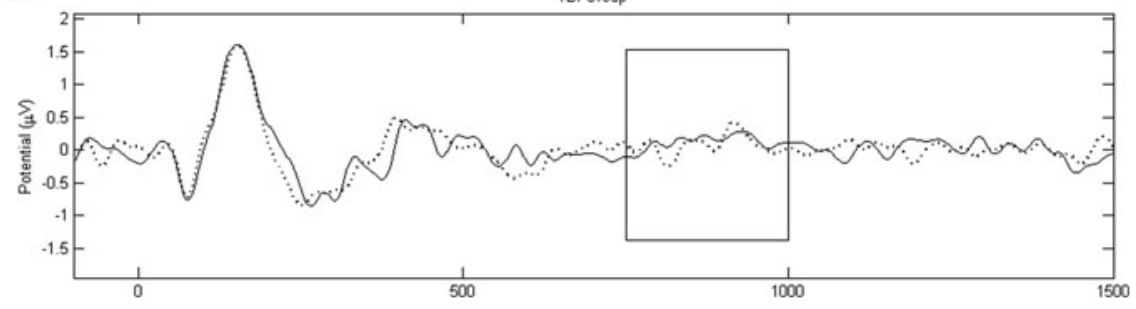

FIG. 1. Group evoke-related potential (ERP) amplitudes $(\mu \mathrm{V})$ at electrode F7 during the Semantic Object Retrieval Test task. Data for the retrieval condition are shown in black; data for the non-retrieval condition are shown by a dashed line. There was a significant difference (750-1000 msec) between conditions for the control group (A) but not the traumatic brain injury (TBI) group (B). 
$(\mathrm{M}=-.12 \mu \mathrm{V}, \mathrm{SD}=.38)$ for the non-retrieval condition $\mathrm{t}(36)=$ $-2.09, p=0.044$. There was no significant difference between groups for the retrieval condition (TBI group $\mathrm{M}=.12 \mu \mathrm{V}, \mathrm{SD}=.54$; $\mathrm{HC}$ group $\mathrm{M}=.25 \mu \mathrm{V}, \mathrm{SD}=.41) \mathrm{t}(36)=.88, p=0.384)$.

\section{Discussion}

We used ERPs to assess aspects of word retrieval in retired professional athletes with a history of TBI. There were no significant behavioral differences in accuracy or reaction time between the TBI group and $\mathrm{HC}$ group. Consistent with previous studies, there was a significant difference in reaction time and accuracy between retrieval and non-retrieval conditions for both groups. ${ }^{15,16}$ For the nonretrieval condition, both groups had longer reaction times and higher accuracy. We postulate non-retrieval trials required a more extensive search through all possible answers before a participant was able to make a final decision, therefore causing longer reaction times. Higher accuracy and longer reaction times for the non-retrieval condition are likely an example of a reaction time-accuracy trade-off.

We found a significant difference between retrieval and nonretrieval conditions in ERP amplitudes for the HC group from $750 \mathrm{msec}$ to $1000 \mathrm{msec}$. The TBI group did not show this differentiation between conditions, suggesting neurophysiological effects associated with previous head trauma.

\section{Use of ERP in the study of word retrieval}

The left fronto-temporal area, specifically the left inferior frontal gyrus and the left posterior middle temporal gyrus, is an integral part of the language circuit. ${ }^{25,26}$ In healthy persons, successful word retrieval elicits different electrical brain activity compared with a nonretrieval condition on the SORT task. In healthy adults, this left frontotemporal difference has been found between $750-1000$ msec. $^{15,16,24}$ We found this same distinction in our HC subjects.

The TBI group showed no significant difference in ERP amplitudes between conditions during the SORT task in the left frontotemporal region, despite preserved performance. This is in contrast with what Chiang and associates ${ }^{16}$ found in their study of $\mathrm{HC}$ subjects and persons with MCI: longer reaction times and differences between retrieval and non-retrieval ERP waveforms in the MCI group. ${ }^{16}$ The word retrieval process requires communication and precise timing between thalamic and cortical regions of the brain. ${ }^{27}$ The absence of ERP differentiation in our TBI cohort could be because of damage to particular brain structures or to deficient communication between necessary brain regions of the semantic retrieval circuit. $^{28}$

One of the long-term effects of repetitive TBI is impaired white matter integrity ${ }^{29-31}$ and decreased thalamo-cortical structural integrity. ${ }^{32}$ Given the compromised white matter integrity found previously in retired professional athletes, ${ }^{29}$ we speculate that reduced structural integrity leads to disrupted coordination of electrical activity between regions, which may lead to discoordination of electrical inputs, reduced synchrony of synaptic and post-synaptic activity, and thus blunted ERP waveform components.

It is interesting to note that the TBI group showed no significant difference in behavioral performance despite evidence of neurological differences in ERP. Thus, either the regions receiving discoordinated electrical signals nonetheless may still be able to function adequately to preserve behavior, or other brain regions that in this setting did not contribute to detectable differences in scalp-recorded ERPs, might be performing a compensatory role. Further studies, evaluating the EEG for frequency band-specific power changes as opposed to ERP, may contribute to clarifying this issue.

In addition to white matter damage, TBI typically also damages gray matter. Gray matter damage has been found to reduce synchronization of active generators, which can lead to reduced EEG amplitude. ${ }^{33}$ While we cannot determine whether the loss of ERP difference in the TBI group is secondary to white matter or gray matter pathology from this study, the findings do support disruption of the retrieval network.

Our previous study of retired NFL players found that players in whom cognitive decline developed with age had deficits in tests of word retrieval (SORT, Boston Naming Test, episodic memory). ${ }^{34}$ In addition, these persons had abnormal cerebral blood flow in the left inferior parietal lobule, left posterior superior gyrus, and left temporal pole. The neuropsychological deficits in retrieval in the group with cognitive decline suggest that the atypical EEG in our normally performing population indicates a risk for later development of difficulties in retrieval processes.

\section{Limits of the study}

This study is limited to male retired NFL players, but similar presentations of TBI could be present in other populations and across sexes. The athletes in the study varied in amount of time since retirement, ranging from five to 50 years. The wide range made it difficult to tease apart effects of TBI recovery over time. There was no significant correlation between years of retirement and average ERP amplitude, however.

The athletes reported a wide number of concussions, ranging between 1 and 20 (including all grades). There were no statistically significant differences between participants with grade 3 concussions and participants without grade 3 concussions, ${ }^{29}$ but our cohort of participants reporting grade 3 concussions is small (12 athletes reported one or more grade 3 ). This study was designed to investigate the effects of multiple TBI and therefore may not generalize to individuals with only one TBI. There is currently no medical diagnostic tool to verify number of previous concussions. Detailed interviews were conducted to ensure athletes understood the definition of concussion to accurately identify TBIs. More detailed records of concussion classification would be helpful to establish how severity and frequency of TBI affects the word retrieval process.

\section{Conclusion}

Retired professional athletes had altered electrical signatures compared with control subjects during the SORT task, suggesting neurophysiological effects of TBI. Further investigating word retrieval networks in persons with TBI can extend our understanding of neural mechanisms of word retrieval deficits and the long-term implications of repeated TBI. Identifying ERP abnormalities associated with impaired word retrieval may be used as a clinical marker for assessment of athletes with TBI. Future studies should examine athletes longitudinally to determine whether changes in amplitude are, in fact, an indication of early stages of impairment.

This study is a first step toward establishing a marker of underlying neuropathology of word retrieval in TBI when there has been no disruption in performance measures. This will only be borne out by longitudinal studies. This notion of a link between this decrease in retrieval ERP and retrieval deficits with aging would be further supported by following these persons to determine whether they become semantically impaired and by marking the time course of the ERP changes and behavioral impairment. 


\section{Acknowledgments}

The authors are grateful for Shradhdha Joshi, Lindy Loneman, Athula Pudhiyidath, and Scott Shakal for their assistance with data collection. A special thank you to Dr. Francesca Filbey for her insightful comments on the early drafts.

\section{Author Disclosure Statement}

No competing financial interests exist.

\section{References}

1. Langlois, J.A., Rutland-Brown, W., and Wald, M.M. (2006). The epidemiology and impact of traumatic brain injury: a brief overview. J. Head Trauma Rehabil. 21, 375-378.

2. Ylvisaker, M. (1986). Language and communication disorders following pediatric head injury. J. Head Trauma Rehabil. 1, 48-56.

3. King, K.A., Hough, M.S., Walker, M.M., Rastatter, M., and Holbert, D. (2006). Mild traumatic brain injury: effects on naming in word retrieval and discourse. Brain Inj. 20, 725-732.

4. McWilliams, J., and Schmitter-Edgecombe, M. (2008). Semantic memory organization during the early stage of recovery from traumatic brain injury. Brain Inj. 22, 243-253.

5. Hart, J., Jr., Maguire, M.J., Motes, M., Mudar, R.A., Chiang, H.S., Womack, K.B., and Kraut, M.A. (2013). Semantic memory retrieval circuit: role of pre-SMA, caudate, and thalamus. Brain Lang. 126, 89-98.

6. Gaetz, M., and Bernstein, D.M. (2001). The current status of electrophysiologic procedures for the assessment of mild traumatic brain injury. J. Head Trauma Rehabil. 16, 386-405.

7. Luck, S.J. (2014). An Introduction to the Event-Related Potential Technique. MIT Press: Cambridge, MA.

8. Thériault, M., De Beaumont, L., Gosselin, N., Filipinni, M., and Lassonde, M. (2009). Electrophysiological abnormalities in well functioning multiple concussed athletes. Brain Inj. 23, 899-906.

9. Gosselin, N., Bottari, C., Chen, J.K., Huntgeburth, S.C., De Beaumont L., Petrides, M., Cheung, B., and Ptito, A. (2012). Evaluating the cognitive consequences of mild traumatic brain injury and concussion by using electrophysiology. Neurosurg. Focus 33, E7.

10. Lachapelle, J., Bolduc-Teasdale, J., Ptito, A., and McKerral, M. (2008). Deficits in complex visual information processing after mild TBI: electrophysiological markers and vocational outcome prognosis. Brain Inj. 22, 265-274.

11. Thériault, M., De Beaumont, L., Tremblay, S., Lassonde, M., and Jolicoeur, P. (2011). Cumulative effects of concussions in athletes revealed by electrophysiological abnormalities on visual working memory. J. Clin. Exp. Neuropsychol. 33, 30-41.

12. Kutas, M., and Federmeier, K.D. (2011). Thirty years and counting: finding meaning in the N400 component of the event related brain potential (ERP). Annu. Rev. Psychol. 62, 621-647.

13. Kraut, M.A., Kremen, S., Segal, J.B., Calhoun, V., Moo, L.R., and Hart J., Jr. (2002). Object activation from features in the semantic system. J. Cogn. Neurosci. 14, 24-36.

14. Calley, C.S., Kraut, M.A., Spence, J.S., Briggs, R.W., Haley, R.W., and Hart J., Jr. (2010). The neuroanatomic correlates of semantic memory deficits in patients with Gulf War illnesses: a pilot study. Brain Imaging Behav. 4, 248-255.

15. Chiang, H.S., Mudar, R.A., Spence, J.S., Pudhiyidath, A., Eroh, J., DeLaRosa, B., Kraut, M.A., and Hart, J., Jr. (2014). Age-related changes in feature-based object memory retrieval as measured by event-related potentials. Biol. Psychol. 100, 106-114.

16. Chiang, H.S., Mudar, R.A., Pudhiyidath, A., Spence, J.S., Womack, K.B., Cullum, C.M., Tanner, J.A., Eroh, J., Kraut, M.A., and Hart, J., Jr. (2015). Altered neural activity during semantic object memory retrieval in amnestic mild cognitive impairment as measured by eventrelated potentials. J. Alzheimers Dis. 46, 703-717.

17. Kraut, M.A., Cherry, B., Pitcock, J.A., Anand, R., Li, J., Vestal, L., Henderson, V.W., and Hart, J., Jr. (2007). The Semantic Object
Retrieval Test (SORT) in amnestic mild cognitive impairment. Cogn. Behav. Neurol. 20, 62-67.

18. Costafreda, S.G., Fu, C.H., Lee, L., Everitt, B., Brammer, M.J., and David, A.S. (2006). A systematic review and quantitative appraisal of fMRI studies of verbal fluency: role of the left inferior frontal gyrus. Hum. Brain Mapp. 27, 799-810.

19. Broca, P. (1861). Remarks on the seat of the faculty of articulated language, following an observation of aphemia (loss of speech). Bulletin de la Société Anatomique 6, 330-357.

20. Delorme, A., and Makeig, S. (2004). EEGLAB: an open source toolbox for analysis of single-trial EEG dynamics including independent component analysis. J. Neurosci. Methods 134, 9-21.

21. Jung, T.P., Makeig, S., Humphries, C., Lee, T.W., McKeown, M.J., Iragui, V. and Sejnowski, T.J. (2000). Removing electroencephalographic artifacts by blind source separation. Psychophysiology 37, 163-178.

22. Bell, A.J., and Sejnowski, T.J. (1995). An information-maximization approach to blind separation and blind deconvolution. Neural Comput. 7, 1129-1159.

23. Junghöfer, M., Elbert, T., Tucker, D.M., and Rockstroh, B. (2000). Statistical control of artifacts in dense array EEG/MEG studies. Psychophysiology 37, 523-532.

24. Brier, M.R., Maguire, M.J., Tillman, G.D., Hart, J., and Kraut, M.A (2008). Event-related potentials in semantic memory retrieval. J. Int. Neuropsychol. Soc. 14, 815-822.

25. Badre, D., and Wagner, A.D. (2002). Semantic retrieval, mnemonic control, and prefrontal cortex. Behav. Cogn. Neurosci. Rev. 1, 206-218.

26. Whitney, C., Kirk, M., O'Sullivan, J., Lambon Ralph, M.A., and Jefferies, E. (2012). Executive semantic processing is underpinned by a large-scale neural network: revealing the contribution of left prefrontal, posterior temporal, and parietal cortex to controlled retrieval and selection using TMS. J. Cogn. Neurosci. 24, 133-147.

27. Hart, J., Jr., (2015). The Neurobiology of Cognition and Behavior. Oxford University Press: New York.

28. Crosson, B. (1999). Subcortical mechanisms in language: lexicalsemantic mechanisms and the thalamus. Brain Cogn. 40, 414-438.

29. Strain, J., Didehbani, N., Cullum, C.M., Mansinghani, S., Conover, H., Kraut, M.A., Hart, J., Jr., and Womack, K.B. (2013). Depressive symptoms and white matter dysfunction in retired NFL players with concussion history. Neurology 81, 25-32.

30. Henry, L.C., Tremblay, J., Tremblay, S., Lee, A., Brun, C., Lepore, N., Theoret, H., Ellemberg, D., and Lassonde, M. (2011). Acute and chronic changes in diffusivity measures after sports concussion. J. Neurotrauma 28, 2049-2059.

31. Koerte, I.K., Ertl-Wagner, B., Reiser, M., Zafonte, R., and Shenton, M.E. (2012). White matter integrity in the brains of professional soccer players without a symptomatic concussion. JAMA 308, 1859-1861.

32. Little, D.M., Kraus, M.F., Joseph, J., Geary, E.K., Susmaras, T., Zhou, X.J., Pliskin, N. and Gorelick, P.B. (2010). Thalamic integrity underlies executive dysfunction in traumatic brain injury. Neurology $74,558-564$

33. Thatcher, R., Biver, C., McAlaster, R., and Salazar, A. (1998). Biophysical linkage between MRI and EEG coherence in closed head injury. Neuroimage 8, 307-326.

34. Hart, J., Kraut, M.A., Womack, K.B., Strain, J., Didehbani, N., Bartz, E., Conover, H., Mansinghani, S., Lu, H., and Cullum, C.M. (2013). Neuroimaging of cognitive dysfunction and depression in aging retired National Football League players: a cross-sectional study. JAMA Neurol. 70, 326-335.

Address correspondence to: Julie M. Fratantoni, MS Center for BrainHealth

The University of Texas at Dallas 2200 West Mockingbird Lane Dallas, TX 75235

E-mail: Julie.Fratantoni@utdallas.edu 